\title{
THERMALIZED KINETIC AND FLUID MODELS FOR REENTRANT SUPPLY CHAINS*
}

\author{
D. ARMBRUSTER ${ }^{\dagger}$ AND C. RINGHOFER ${ }^{\dagger}$
}

\begin{abstract}
Standard stochastic models for supply chains predict the throughput time (TPT) of a part from a statistical distribution, which is dependent on the work in progress at the time the part enters the system. So they try to predict a transient response from data which are sampled in a quasi-steady-state situation. For reentrant supply chains this prediction is based on insufficient information, since subsequent arrivals can dramatically change the TPT. This paper extends these standard models by introducing the concept of a stochastic phase velocity which dynamically updates the TPT estimate. This leads to the concepts of temperature and diffusion in the corresponding kinetic and fluid models for supply chains.
\end{abstract}

Key words. reentrant supply chains, traffic flow models, Boltzmann equation, ChapmanEnskog, fluid limits

AMS subject classifications. 65 N35, 65 N05

DOI. $10.1137 / 030601636$

1. Introduction. This paper is concerned with models for supply chains with reentrant nodes and their macroscopic, i.e., long term, limits, resulting in a modification of standard discrete event simulation algorithms. Generally, a supply chain is a network of suppliers who produce goods, both for one another and for a generic customer. Goods travel from origin (suppliers) to destination (customers), visiting intermediate suppliers and being altered in the process. Conservation rules at each supplier define its outputs as functions of its inputs [6]. In discrete event simulation each item to be produced is modeled individually; i.e., the arrival time at a certain supplier is recorded, a processing time (throughput time (TPT)) is chosen randomly from a given distribution, and the item is passed to the next supplier after the TPT has elapsed.

The term supply chain is rather generic in the sense that the above definitions can apply to a wide variety of situations. The individual suppliers can be machines on a factory floor, in which case the distribution of processing times is given by such quantities as mean time to failure, mean time to scheduled maintenance, etc. On the other hand, the supply chain can model the whole evolution of a product from the actual production process to quality control, packaging, and shipping to distributors. In this case individual suppliers consist of whole factories, and TPT distributions are given by experimental data or more detailed discrete event simulations of the individual suppliers in the chain. In either case the standard mathematical model is the following. We consider a chain of $M$ suppliers $S_{1}, \ldots, S_{M}$ and denote the time at which the lot number $n$ arrives at supplier $S_{m}$ by $a_{n}^{m}$ and the time it exits supplier $S_{m}$ (and arrives at $S_{m+1}$ ) by $e_{n}^{m}$. The arrival and exit times are computed via the law
(a) $e_{n}^{m}=a_{n}^{m}+\tau_{n}^{m}$,
(b) $d \mathcal{P}\left\{\tau_{n}^{m}=r\right\}=\mathcal{T}_{m}\left(r, a_{n}^{m}\right) d r$
(c) $a_{n}^{m+1}=e_{n}^{m}$.

* Received by the editors November 3, 2003; accepted for publication (in revised form) June 28, 2004; published electronically DATE. This work was supported by the NSF under grant DMS0204543.

http://www.siam.org/journals/mms/x-x/60163.html

${ }^{\dagger}$ Department of Mathematics, Arizona State University, Tempe, AZ 85287-1804 (armbruster@ asu.edu,ringhofer@asu.edu). 
Here $\mathcal{T}_{m}(r, a)$ denotes the time-dependent distribution of TPTs of supplier $S_{m}$. So $\tau_{n}^{m}$ is chosen randomly according to $(1.1)(\mathrm{b})$. The TPT distribution $\mathcal{T}_{m}$ is usually dependent on the total number $W_{m}(t)$ of lots handled by the supplier $S_{m}$ at time $t$, the so-called work in progress (WIP). $W_{m}(t)$ is given by

$$
W_{m}(t)=\sum_{n} H\left(t-a_{n}^{m}\right)-H\left(t-a_{n}^{m+1}\right),
$$

where $H$ denotes the usual Heaviside function and the TPT distribution $\mathcal{T}_{m}$ in (1.1) is taken to be $\mathcal{T}_{m}=\mathcal{T}_{m}\left(r, W_{m}\left(a_{n}^{m}\right)\right)$.

For instance, in the case when supplier $S_{m}$ models a single machine with a buffer queue, the TPT distribution would be given by $\mathcal{T}_{m}\left(r, a_{n}^{m}\right)=\left(1+W_{m}\left(a_{n}^{m}\right)\right) P(r)$, with $P(r)$ the probability distribution of the processing time; i.e., the TPT is computed from the number of lots in front of lot $n$ times the random time it takes to process one lot. In the case of complicated supply chains, where each individual supplier models a whole factory, the dependence of the distribution $\mathcal{T}_{m}$ on the WIP $W_{m}$ is more complex and given by experimental data.

Continuous product models. If the number of lots considered in a given time interval is very large, it is computationally preferable to replace the model (1.1) by a model where the WIP $W_{m}(t)$ is a continuous function. As a further consequence, this leads to a class of models often referred to as fluid models in the supply chain literature [1], [8]. In this paper we will refer to them as continuous product models so as not to confuse them with the model developed in section 4 . In the continuous product model the primary variables are $W_{m}(t)$ and the fluxes $\lambda_{m}(t)$ from supplier $S_{m-1}$ to supplier $S_{m}$. A random TPT function $\tau(t)$ is computed from the WIP as before. The WIP $W_{m}(t)$ is then given by the conservation law

$$
\frac{d}{d t} W_{m}(t)=\lambda_{m}(t)-\mu_{m}(t), \quad \lambda_{m+1}(t)=\mu_{m}(t)
$$

and the fluxes are given, according to (1.1), by

$$
\mu_{m}(t)=\int \delta(s+\tau(s)-t) \lambda_{m}(s) d s
$$

The flux model (1.2)-(1.3) coincides with (1.1) if the fluxes $\lambda_{m}$ are taken to be concentrated at the arrival times, i.e., for $\lambda_{m}(t)=\sum_{n} \delta\left(t-a_{n}^{m}\right)$. However, for a large number of lots the fluxes $\lambda_{m}$ are replaced by continuous functions.

In this paper we are concerned with the second type of situation, where a node in the supply chain models a more complex entity. In particular, we are interested in the case of reentrant supplier nodes. So the supplier $S_{m}$ actually consists of a complex system of subnodes which have to be passed by a given lot repeatedly. We are not able to model the whole complex system of subnodes on the scale of our simulation but replace it by a probability distribution for the TPT dependent on the WIP. This results in the following inadequacy of the model (1.1). According to the formula (1.1), the TPT is determined by the state of the supplier $S_{m}$ at the time $a_{n}^{m}$, when lot number $n$ arrives. If supplier $S_{m}$ is a reentrant system itself, then the lots arriving after time $a_{n}^{m}$ will significantly influence the TPT $\tau_{n}^{m}$ since they will compete with lot number $n$ in the individual subqueues of the system. This competition will be governed by a given set of policies used by supplier $S_{m}$, such as FIFO, PUSH, or PULL [13], which we assume to be given. In particular, it is observed experimentally that for reentrant suppliers there exists a critical load, i.e., a critical value of $W_{m}$ 
beyond which the TPT $\tau_{n}^{m}$ grows rapidly and actually may develop a pole [2]. It is therefore inadequate to compute $\tau_{n}^{m}$ solely based on information available at time $a_{n}^{m}$. This problem will of course be relevant only in situations where the WIP $W_{m}$, and therefore the distribution $\mathcal{T}_{m}$, changes significantly in the time interval $\left[a_{n}^{m}, a_{n}^{m+1}\right]$.

It should be mentioned that one often considers more complex supply networks instead of supply chains, that is, systems where the product flow is more complex than just a linear flow from one supplier to the next. This additional complication is not relevant for the subject of this paper since we will be concerned only with an improved stochastic model for one individual supplier. So the results of this paper apply in the same way to supply networks, with the obviously more complex notation introduced by the topology of the network. Also, not all suppliers in a chain or network will be of a reentrant nature, and the work presented in this paper concerns only the reentrant nodes of the network.

The basic idea of this paper is the following. Instead of modeling the supplier $S_{m}$ as a simple machine with a buffer queue, as given by the formula (1.1), we model supplier $S_{m}$ as a machine working at a variable speed, which is given (randomly) by a distribution depending on the WIP $W_{m}(t)$ as a function of time. Thus, later arrivals influence the TPT of a given lot by influencing the speed with which the whole supplier node $S_{m}$ is able to process. We introduce this concept of a variable speed by introducing a virtual time and a phase for the supplier $S_{m}$. In this virtual time the processing time $\tau_{n}^{m}$ is constant, and we vary instead the transformation from the virtual to the real time, i.e., the phase, randomly. We refer to this concept as the "random phase" approach, which is presented in section 2. This random phase approach results in a stochastic model which is formally equivalent to a Monte Carlo discretization of a Boltzmann equation. In section 3 we derive the corresponding kinetic evolution equation for the probability density, resulting in a so-called traffic flow model [9], [10] for the parts in the system. In section 4 we derive a macroscopic, i.e., lower-dimensional, model for the expectations of the flux through the system for large time scales via a Chapman-Enskog expansion [4]. Finally, in section 5 we present some numerical results, comparing the standard discrete event simulation model (1.1) to the random phase approach and verifying the asymptotic validity of the fluid model for the expectations.

2. The random phase model. In this section we present the basic concept of modeling the supply chain node $S_{m}$ as a "machine" operating at a variable, randomly chosen, speed. This accounts for the fact that in a reentrant system later arrivals influence the processing time of a particular lot.

2.1. The random phase. We start with the deterministic situation. Assume that at any given time $t$ the processing time $\tau$ in (1.1) is given by a function $\tau=T(t)$. Since we are, for the rest of this paper, concerned only with one node, we will suppress the node index $m$ from here on. The key idea is to interpret the quantity $\frac{1}{\tau}$ as a phase velocity. We introduce the phase $s$ for the given node in the supply chain as the antiderivative of the frequency, $\frac{1}{T}$, i.e., by

$$
\text { (a) } s=\phi(t), \quad\left(\text { b) } \frac{d \phi}{d t}=\frac{1}{T(t)}, \quad \phi(0)=0 .\right.
$$

Here we assume that we start the simulation at time $t=0$. Note that the transformation (2.1) can be interpreted as a "virtual time." In this virtual time $s$, given by the phase, the processing time is taken to be constantly equal to 1 . So for lot number $n$, 
the exit time $e_{n}$ is computed from the arrival time $a_{n}$ via the relation

$$
\phi\left(e_{n}\right)=\phi\left(a_{n}\right)+1, \quad e_{n}=\phi^{-1}\left(1+\phi\left(a_{n}\right)\right) .
$$

Obviously, for constant processing times $T(t)=T_{0},(2.2)$ reduces to $e_{n}=a_{n}+T_{0}$, which coincides with (1.1). For time variant but deterministic TPTs the exit time $e_{n}$ would be given implicitly by

$$
\int_{a_{n}}^{e_{n}} \frac{d t}{T(t)}=1
$$

In the case that the processing times $\tau_{n}$ are computed randomly from a time-dependent distribution, $(2.1)(\mathrm{b})$ is replaced by updating the function $\phi(t)$ in certain time intervals. So $(2.1)$ (b) becomes

$$
\phi\left(t+\frac{1}{\omega}\right)=\phi(t)+\frac{1}{\omega \tau(t)}, \quad \phi(0)=0, \quad d \mathcal{P}\{\tau(t)=r\}=\mathcal{T}(r, t) d r .
$$

So, as in (1.1), the processing times are chosen randomly from a given distribution $\mathcal{T}(r, t)$. The difference now lies in the fact that the exit time $e_{n}$ in $(2.2)$ is computed from the whole time evolution of the processing times $\tau(t)$ and not only by their value at the arrival time $a_{n}$. In the deterministic case, where the probability density $\mathcal{T}(r, t)$ is concentrated at one point in $r,(2.3)$ becomes the discretization of (2.1)(b) by Euler's method with a time step $\frac{1}{\omega}$. $\omega$, the update frequency for the virtual time $s$, will be a key parameter to be chosen later.

We will modify the algorithm (2.3) somewhat for the following reason. For analytic purposes, we would prefer to have a continuous time model, where the function $\phi(t)$ is not given only at discrete time intervals. This could be achieved by the limit $\omega \rightarrow \infty$, giving a stochastic process. However, in this limit we would lose the information about $\omega$ which will be a key parameter controlling the stochasticity of the model. So we take a slightly different approach. Instead of updating the phase velocity $\frac{1}{\tau}$ with probability 1 every time step of length $\frac{1}{\omega}$, we update the phase velocity every arbitrary time step $\Delta t$ with probability $\omega \Delta t$. This gives the algorithm

$$
\text { (a) } \phi(t+\Delta t)=\phi(t)+\frac{\Delta t}{\eta(t)}, \quad \eta(t+\Delta t)=\kappa(t) \tau(t)+(1-\kappa(t)) \eta(t),
$$

(b) $\mathcal{P}\{\kappa(t)=1\}=\omega \Delta t, \quad \mathcal{P}\{\kappa(t)=0\}=1-\omega \Delta t, \quad d \mathcal{P}\{\tau(t)=r\}=\mathcal{T}(r, t) d r$,

$$
\text { (c) } \phi(0)=0, \quad d \mathcal{P}\{\eta(0)=r\}=\mathcal{T}(r, 0) d r .
$$

So every $\Delta t$ we choose a random number $\kappa$, which is either zero or one, to decide whether to update the TPT $\eta$. Clearly, (2.4) reduces to (2.3) for $\Delta t=\frac{1}{\omega}$ but has the advantage that an arbitrary time step $\Delta t<\frac{1}{\omega}$ can be used, which will allow us to carry out the limit $\Delta t \rightarrow 0$. We will in general assume a variable and adaptive update frequency (or time step in (2.3)) and let $\omega$ depend on $\eta(t)$ and the time $t$ as well. So $(2.4)$ (b) will be replaced by

$$
\begin{gathered}
\mathcal{P}\{\kappa(t)=1\}=\Delta t \omega(\eta(t), t), \quad \mathcal{P}\{\kappa(t)=0\}=1-\Delta t \omega(\eta(t), t), \\
d \mathcal{P}\{\tau(t)=r\}=\mathcal{T}(r, t) d r .
\end{gathered}
$$

The remaining question is how to choose the update frequency $\omega$. We will deal with this question in section 3 . 
2.2. Discrete event and continuous product algorithms. Based on the evolution equation (2.4) for the virtual time $\phi(t)$ the discrete event simulation algorithm now proceeds as follows. For lot number $n$, arriving at time $a_{n}$,

- at time $a_{n}$ increase the WIP $W(t)$ by one and adjust the probability distribution $\mathcal{T}(r, t)$ accordingly;

- record $\phi\left(a_{n}\right)$, keep evolving $\phi(t)$ according to (2.4), and wait until $\phi(t)$ reaches the value $\phi\left(a_{n}\right)+1$;

- record the exit time $e_{n}=\phi^{-1}\left(1+\phi\left(a_{n}\right)\right)$ and set this equal to the arrival time of lot $n$ in the next node of the supply chain;

- at time $e_{n}$ reduce the WIP $W(t)$ by one and adjust the probability distribution $\mathcal{T}(r, t)$ again.

The above algorithm is slightly more complicated than the one given by (1.1) since the exit time $e_{n}$ is not known a priori, and therefore we have to check for each lot in the node $S$ at each time step whether $\phi(t)$ has reached the value $\phi\left(a_{n}\right)+1$. It renders itself, however, very easily to a discrete time-continuous product model, similar to the one given in (1.2)-(1.3). In this type of model the individual lots are replaced by a continuous quantity, and the primary variables become the WIP $W(t)$ and the fluxes $\lambda(t)$ and $\mu(t)$ in and out of the node $S$. The evolution of the WIP $W_{m}(t)$ is then governed by the conservation law $\frac{d}{d t} W(t)=\lambda(t)-\mu(t)$ or, using discrete time steps,

$$
W(t+\Delta t)=W(t)+\Delta t[\lambda(t)-\mu(t)] .
$$

So the question becomes how to compute the outflux $\mu(t)$ in terms of the influx $\lambda(t)$. In the case of discrete events the fluxes are given by superpositions of $\delta$-functions according to

$$
\mu(t)=\sum_{n} \delta\left(t-e_{n}\right)=\sum_{n} \delta\left(t-\phi^{-1}\left(\phi\left(a_{n}\right)+1\right)\right)=\int \delta\left(t-\phi^{-1}\left(\phi\left(t^{\prime}\right)+1\right)\right) \lambda\left(t^{\prime}\right) d t^{\prime}
$$

with the discrete influx function $\lambda(t)$ given by $\lambda(t)=\sum_{n} \delta\left(t-a_{n}\right)$. Changing variables in (2.6) and using $\frac{d}{d t} \phi=\frac{1}{\eta}$ (where $\phi$ is piecewise linear and $\eta$ is piecewise constant in time intervals of length $\Delta t$ according to (2.4)) gives

$$
\mu(t)=\frac{\eta\left(\phi^{-1}(\phi(t)-1)\right)}{\eta(t)} \lambda\left(\phi^{-1}(\phi(t)-1)\right)
$$

As in (1.2)-(1.3), the continuous flux formulation now consists of replacing the concentrated influx function $\lambda(t)$ by a continuous function and computing the outflux $\mu$ according to (2.7) using the random phase function $\phi$ and its inverse. It should be pointed out that any realization of the random phase function $\phi$ is always monotone, and therefore its inverse always exists. Equation (2.7) implies that a record of the influx $\lambda$ and the phase speed $\eta$ in (2.4) has to be kept for some time such that they can be evaluated at time $t^{\prime}=\phi^{-1}(\phi(t)-1)$ by interpolation. In contrast, using the same update algorithm for the TPTs, the flux model (1.3) corresponding to the original discrete event simulator would (after the obvious change in notation) read

$$
\mu(t)=\int \delta(s+\eta(s)-t) \lambda(s) d s
$$


3. A kinetic model. The update algorithm (2.4) is reminiscent of a Monte Carlo method for the solution of a Boltzmann equation (see [5] for an introduction). The phase function $\phi(t)$ plays the role of a position which is advanced with a randomly changing velocity $\frac{1}{\eta(t)}$. It is therefore tempting to derive a kinetic formulation for the evolution of the lots whose flux is given by the algorithm (2.2). In the context of supply chains, these models are often referred to as traffic flow models [3], [10], because of the analogy to lots moving on a freeway from start to completion. The basic idea is the following. We introduce an artificial, dimensionless variable $x$, called the degree of completion (DOC), which varies between $x=0$ and $x=1$. A lot arrives at time $t=a$ and moves from $x=0$ to $x=1$ along a trajectory with velocity $\frac{1}{\eta_{a}(t)}$ in such a way that it reaches the end $x=1$ at time $t=e$; i.e., the velocity has to be chosen in such a way that

$$
\int_{a}^{e} \frac{1}{\eta_{a}(t)} d t=1
$$

holds. For instance, in the kinetic model corresponding to (1.1), one would choose $\eta_{a}(t)=\tau(a)$, with $\tau$ generated randomly at time $t=a$, since the exit time is determined at $t=a$, and therefore a constant velocity can be used. We now derive the kinetic model corresponding to the random phase approach from section 2. Again, as in the derivation of the flux model in (2.7), we proceed by deriving the kinetic model in virtual time. Denoting the position of the lot at time $t$ by $\xi_{a}(t)$, we define the position in virtual time by $\tilde{\xi}_{a}(s)=\xi_{a}\left(\phi^{-1}(s)\right)$. Since the TPT in the virtual time is equal to unity, $\tilde{\xi}_{a}(s)$ moves with a constant speed equal to one, giving

$$
\begin{gathered}
\tilde{\xi}_{a}(s+\Delta s)=\tilde{\xi}_{a}(s)+\Delta s \quad \text { or } \quad \xi_{a}(t+\Delta t)=\xi_{a}(t)+\Delta s \\
s=\phi(t), \quad \Delta s=\phi(t+\Delta t)-\phi(t)=\frac{\Delta t}{\eta_{a}(t)}
\end{gathered}
$$

according to (2.4)(a). This gives the particle model, together with the phase speed model (2.4),

$$
\text { (a) } \xi_{a}(t+\Delta t)=\xi_{a}(t)+\frac{\Delta t}{\eta_{a}(t)}, \quad \eta_{a}(t+\Delta t)=\kappa(t) \tau(t)+(1-\kappa(t)) \eta_{a}(t),
$$

(b) $\mathcal{P}\{\kappa(t)=1\}=\omega \Delta t, \quad \mathcal{P}\{\kappa(t)=0\}=1-\omega \Delta t, \quad d \mathcal{P}\{\tau(t)=r\}=\mathcal{T}(r, t) d r$,

$$
\text { (c) } \xi_{a}(a)=0, \quad d \mathcal{P}\left\{\eta_{a}(a)=r\right\}=\mathcal{T}(r, a) d r
$$

for the evolution of the position $\xi_{a}$ of the lot arriving at $t=a$. The WIP and the flux at any point $x \in[0,1]$, in particular the influx and outflux $\lambda$ and $\mu$ at $x=0$ and $x=1$, would be given by

$$
W(t)=\int\left[H\left(\xi_{a}(t)\right)-H\left(\xi_{a}(t)-1\right)\right] \lambda(a) d a, \quad F(x, t)=\int \eta_{a}(t) \delta\left(x-\xi_{a}(t)\right) \lambda(a) d a,
$$

where $\lambda(a)$ is concentrated on the arrival times $a_{n}$ for a discrete model or is a continuous function for a continuous product model. In either case, we assume that $a>0$ holds for all arrival times, and therefore $\lambda(a)=0$ holds for $a<0$. Note that the 
algorithm (3.1) for advancing the lot trajectory $\xi_{n}$ is essentially the same as the algorithm (2.4) for advancing the phase function, and the difference lies only in the initial condition $(3.1)(\mathrm{c})$.

The mean field assumption. For the rest of this section we will make a mean field assumption; that is, we will assume that the TPT distribution $\mathcal{T}(r, t)$ is a given function. In reality, the TPT distribution will depend on the lot trajectories $\xi$ through the WIP $W$, given by (3.2). The idea underlying this assumption is that, for many lots present in the system, the impact of one individual lot on the distribution $\mathcal{T}$ is negligible. Therefore, the lots can be treated as approximately statistically independent. This is a standard approach for a system of many molecules in gas dynamics [5]. Making this mean field assumption, we have the following theorem.

TheOrem T1. Let $f_{a}(x, r, t)$ denote the probability density for finding $\xi_{a}, \eta_{a}$, defined by (3.1), at $x$ and $r$, i.e., $d \mathcal{P}\left\{\xi_{a}(t)=x, \eta_{a}(t)=r\right\}=f_{a}(x, r, t) d x d r$. Then $f_{a}$ satisfies the initial value problem

$$
\text { (a) } \partial_{t} f_{a}+\frac{1}{r} \partial_{x} f_{a}=\mathcal{T}(r, t) \int \omega\left(r^{\prime}, t\right) f_{a}\left(x, r^{\prime}, t\right) d r^{\prime}-\omega(r, t) f_{a}(x, r, t), \quad t>a,
$$

(b) $f_{a}(x, r, a)=\delta(x) \mathcal{T}(r, a)$

in the limit $\Delta t \rightarrow 0$, weakly in $x, r$, and $t$.

The proof of Theorem T1 is deferred to section 7 .

Using Theorem $\mathrm{T} 1$, we now derive the corresponding initial boundary value problem for the kinetic density $f(x, r, t)$ of the number of lots at position $x$ with velocity $\frac{1}{r}$. We define $f$ by

$$
f(x, r, t)=\int H(t-a) f_{a}(x, r, t) \lambda(a) d a .
$$

$f$ is not a probability density since the trajectory $\xi_{a}$ will exist only for $t>a$, and the number of lots will not be constant over time. The lot density $f$ will satisfy the same Boltzmann equation as the probability density $f_{a}$ with a more natural influx boundary condition replacing the initial condition (3.3)(b). We have the following theorem.

THEOREM T2. The number density (3.4) satisfies the initial boundary value problem

$$
\text { (a) } \partial_{t} f+\frac{1}{r} \partial_{x} f=\mathcal{T}(r, t) \int \omega\left(r^{\prime}, t\right) f\left(x, r^{\prime}, t\right) d r^{\prime}-\omega(r, t) f(x, r, t), \quad x>0, t>0 \text {, }
$$

(b) $f(0, r, t)=r \lambda(t) \mathcal{T}(r, t), \quad f(x, r, 0)=0$,

weakly in $x, r$, and $t$.

The proof of Theorem T2 is deferred to section 7 .

Remark. The Boltzmann equation (3.5) is a generalization of the kinetic model derived in a previous work [3]. The random phase approach has introduced the concept of collisions, i.e., the integral operator on the right-hand side of (3.5), into the particle model. This in turn defines the equivalent of a "temperature," given by the variance of the equilibrium distribution $\frac{\mathcal{T}}{\omega}$. In the next section we will analyze the thermalized regime, where the flow is actually collision dominated. 
It remains to choose the update frequency $\omega(r, t)$. We remark that there necessarily is some arbitrariness in this choice. The discrete event simulation model (1.1) yields one particular realization of a stochastic process. To compute meaningful answers, one therefore will run a series of discrete event experiments and compute averages. How many experiments one uses will determine how close the result will be to the average, where a degenerate TPT distribution (a $\delta$-function concentrated at the mean) is used. So the size of $\omega$ has to be chosen in the same way the number of realizations of the discrete event simulation model is chosen. The guiding principle here has to be that the overall number of random numbers generated is the same. As to the precise form of the update frequency $\omega(r, t)$ in $(2.4)$ and $(3.1)$, the result of Theorem T2 gives an indication of how to proceed. The basic idea of the approach presented so far was to modify the discrete event simulation model (1.1) in order to take into account transient changes in TPTs and influx, which in the case of reentrant nodes will influence the outflux. So, on the other hand, if there are no changes in influx and TPT, the discrete event model (1.1) should correspond to a solution of the Boltzmann equation (3.5). Translating the discrete event simulation (1.1) into a kinetic model along the lines of the approach in [3] gives the following picture. For an arrival time $a$ we pick a TPT $\tau$ according to the distribution $\mathcal{T}(\tau, a)$ and move with the constant velocity $\eta=\frac{1}{\tau}$ until reaching $x=1$ at time $t=a+\tau$. This gives for the lot trajectory in phase space the relations

$$
\xi_{a}(t)=\frac{t-a}{\tau}, \quad \eta_{a}(t)=\tau, \quad d \mathcal{P}\{\tau=r\}=\mathcal{T}(r, a) d r .
$$

We denote by $f_{a}^{d}(x, r, t)$ the corresponding probability density in phase space, i.e., $f_{a}^{d}(x, r, t) d x d r=d \mathcal{P}\left\{\xi_{a}(t)=x, \eta_{a}(t)=r\right\} . f_{a}^{d}$ can be computed exactly from (3.6) as

$$
f_{a}^{d}(x, r, t)=\delta\left(\frac{t-a}{r}-x\right) \mathcal{T}(r, a) .
$$

The number density $f^{d}(x, r, t)$ corresponding to $(3.4)$ is then given by

$f^{d}(x, r, t)=\int H(t-a) \delta\left(\frac{t-a}{r}-x\right) \mathcal{T}(r, a) \lambda(a) d a=r H(x) \mathcal{T}(r, t-r x) \lambda(t-r x)$.

For the random phase model to be equivalent to the discrete event simulation model (1.1), $f^{d}$ has to be a solution of the initial boundary value problem (3.5). Since $f^{d}$ is a function of $t-r x$ it satisfies $\partial_{t} f^{d}+\frac{1}{r} \partial_{x} f^{d}=0 . f^{d}$ also satisfies the boundary condition $(3.5)(\mathrm{b})$. Therefore the residual obtained from inserting $f^{d}$ into (3.5) is given by the collision term

$$
\begin{aligned}
Q\left[f^{d}\right](x, r, t):= & \mathcal{T}(r, t) \int \omega\left(r^{\prime}, t\right) r^{\prime} \mathcal{T}\left(r^{\prime}, t-r^{\prime} x\right) \lambda\left(t-r^{\prime} x\right) d r^{\prime} \\
& -\omega(r, t) r \mathcal{T}(r, t-r x) \lambda(t-r x)
\end{aligned}
$$

and $Q\left[f^{d}\right]=0$ has to hold in the case that neither $\mathcal{T}$ nor $\lambda$ depends on time, giving

$$
0=\mathcal{T}(r) \int \omega\left(r^{\prime}, t\right) r^{\prime} \mathcal{T}\left(r^{\prime}\right) d r^{\prime}-\omega(r, t) r \mathcal{T}(r)
$$

which implies $\omega(r, t)=\frac{\gamma(t)}{r}$ for some arbitrary function $\gamma(t)$. This makes sense since we update the phase speed more frequently for shorter current TPTs, i.e., for a faster 
transient response of the system. So, with this choice, the discrete event simulation (1.1) and the random phase model (2.4) are equivalent for constant $\mathcal{T}$ and $\lambda$ and any function $\gamma(t)$. In the general case, we choose $\gamma(t)$ such that we roughly compute the same number of random numbers for either model. Using the discrete event simulation we pick $\Delta t \lambda(t)$ random numbers in the time interval $\Delta t$. Using the random phase model, we pick $\gamma(t) \int \frac{1}{r} \mathcal{T}(r, t) d r$ in the same time interval. Therefore we set the update frequency $\omega(r, t)$ to

$$
\omega(r, t)=\frac{\lambda(t)}{r T_{-1}}, \quad T_{-1}:=\int \frac{1}{r} \mathcal{T}(r, t) d r .
$$

4. Fluid limits and the Chapman-Enskog expansion. As mentioned before, we do not suggest solving the Boltzmann equation (3.5) for actual simulations. Instead, we will use large time asymptotics to derive fluid dynamic approximations to the solution of the Boltzmann equation, which yield rather attractive simulation tools. The essential asymptotic tool will be the Chapman-Enskog expansion [4] of the Boltzmann equation for a regime where the average TPT and the time intervals between velocity updates in the random phase are short compared to the overall time scale. Fluid equations for the simulation of TPTs have been studied in [2] and [3], leading to simple convection equations for the density of lots as a function of DOC. We essentially recover these results but obtain as a generalization an additional diffusive term arising from the variance of the TPT distribution $\mathcal{T}$. A fluid dynamic approximation to the Boltzmann equation (3.5) is obtained by taking integrals with respect to the TPT variable $r$. Defining the density $\rho(x, t)$ and the flux $F(x, t)$ by

$$
\rho(x, t)=\int f(x, r, t) d r, \quad F(x, t)=\int \frac{1}{r} f(x, r, t) d r,
$$

we obtain the conservation law

$$
\text { (a) } \partial_{t} \rho+\partial_{x} F=0, \quad \text { (b) } \rho(x, 0)=0, \quad \text { (c) } F(0, t)=\lambda(t) .
$$

$\rho$ and $F$, defined by (4.1), represent expectation values of the position and flux densities. The goal is now to find an expression for the flux $F$ in terms of the density $\rho$ using an asymptotic solution of the Boltzmann equation (3.5) for large time scales.

We start by scaling the involved probability distributions and by bringing the Boltzmann equation (3.5) into an appropriate dimensionless form. To this effect we choose some scales for the evolution time $t$ and the TPT $\tau, t_{0}$, and $r_{0}$ (to be determined below), and we define the scaled and dimensionless evolution time and TPT by $t=t_{0} t_{s}$ and $\tau=r_{0} \tau_{s}$. We denote by $T_{1}(t)$ and $\left(\sigma_{\mathcal{T}}(t)\right)^{2}$ the mean and variance of the distributions $\mathcal{T}(r, t)$. We define the scaled TPT and influx by $\mathcal{T}_{s}(r, t)$ and $\lambda_{s}(t)$ by

$$
\mathcal{T}(r, t)=\frac{1}{\sigma_{\mathcal{T}}^{0}} \mathcal{T}_{s}\left(\frac{r}{\sigma_{\mathcal{T}}^{0}}, t_{s}\right), \quad \lambda(t)=\lambda_{0} \lambda_{s}\left(t_{s}\right)
$$

with $\sigma_{\mathcal{T}}^{0}$ some characteristic value for the variance $\sigma_{\mathcal{T}}(t)$. The scaled TPT distribution $\mathcal{T}_{s}$ now has moments and variance

$$
T_{1 s}\left(t_{s}\right)=\frac{T_{1}(t)}{\sigma_{\mathcal{T}}^{0}}, \quad T_{-1 s}\left(t_{s}\right)=\sigma_{\mathcal{T}}^{0} T_{-1}(t), \quad\left(\sigma_{\mathcal{T} s}(t)\right)^{2}=\left(\frac{\sigma_{\mathcal{T}}(t)}{\sigma_{\mathcal{T}}^{0}}\right)^{2}=O(1) .
$$


For this section we will assume that the update frequency $\omega(r, t)$ and the initial value for the phase speed are chosen according to (3.7) as

$$
\omega(r, t)=\frac{\lambda_{0} \sigma_{\mathcal{T}}^{0} \lambda_{s}\left(t_{s}\right)}{r T_{-1 s}} .
$$

We define the scaled solution of (3.5) by $f(x, r, t)=\lambda_{0} f_{s}\left(x, r_{s}, t_{s}\right)$. The scaled and dimensionless version of the Boltzmann equation (3.5) now reads

$$
\begin{aligned}
& \text { (a) } \partial_{t_{s}} f_{s}+\frac{t_{0}}{r_{0}} \frac{1}{r_{s}} \partial_{x} f_{s} \\
& =\frac{\lambda_{0} t_{0} \sigma_{\mathcal{T}}^{0}}{r_{0}} \frac{\lambda_{s}}{T_{-1 s}}\left[\frac{r_{0}}{\sigma_{\mathcal{T}}^{0}} \mathcal{T}_{s}\left(\frac{r_{0}}{\sigma_{\mathcal{T}}^{0}} r_{s}, t_{s}\right) \int \frac{1}{r^{\prime}} f_{s}\left(x, r^{\prime}, t_{s}\right) d r^{\prime}-\frac{1}{r_{s}} f_{s}\left(x, r_{s}, t_{s}\right)\right] \\
& \begin{array}{l}
\text { (b) } f_{s}(x, r, 0)=0, \quad\left(\text { c) } f_{s}\left(0, r, t_{s}\right)=r \lambda_{s}\left(t_{s}\right) \frac{r_{0}}{\sigma_{\mathcal{T}}^{0}} \mathcal{T}_{s}\left(\frac{r_{0} r}{\sigma_{\mathcal{T}}^{0}}, t_{s}\right)\right. \\
\text { (d) } T_{1 s}\left(t_{s}\right):=\int r \mathcal{T}_{s}\left(r, t_{s}\right) d r, \quad T_{-1 s}\left(t_{s}\right):=\int \frac{1}{r} \mathcal{T}_{s}\left(r, t_{s}\right) d r
\end{array}
\end{aligned}
$$

Defining the scaled density and flux in (4.2) by $\rho(x, t)=r_{0} \lambda_{0} \rho_{s}\left(x, t_{s}\right), F(x, t)=$ $\lambda_{0} F_{s}\left(x, t_{s}\right)$, we obtain

$$
\text { (a) } \partial_{t_{s}} \rho_{s}+\frac{t_{0}}{r_{0}} \partial_{x} F_{s}=0, \quad \text { (b) } \rho_{s}(x, 0)=0, \quad \text { (c) } F\left(0, t_{s}\right)=\lambda\left(t_{s}\right) .
$$

There are three time scales involved in this problem, namely

- the scale of the TPTs, given by $\sigma_{\mathcal{T}}^{0}$;

- the overall time scale $r_{0}$ induced by the convection speed $\frac{1}{r_{0}}$;

- the scale of the phase speed updates relative to the overall time scale, given by $\frac{r_{0}}{\lambda_{0} \sigma_{\mathcal{T}}^{0} t_{0}}$.

In order to obtain an equilibrium distribution which varies on an $O(1)$ velocity scale, we set $r_{0}=\sigma_{\mathcal{T}}^{0}$. Setting $t_{0}=r_{0}=\sigma_{\mathcal{T}}^{0}$ makes the convection speed of the problem equal to unity. We will assume that the updates of the phase speed happen very frequently on the time scale of the TPT, i.e., that $\lambda_{0} \sigma_{\mathcal{T}}^{0}=: \frac{1}{\varepsilon} \gg 1$ holds. This is consistent with the regime considered in this paper, where the WIP $W(t)$ changes significantly on the scale of the TPT. This gives, dropping the subscript $s$ for the scaled variables from here on,

$$
\text { (a) } \partial_{t_{s}} f_{s}+\frac{1}{r_{s}} \partial_{x} f_{s}=\frac{\lambda_{s}}{\varepsilon T_{-1 s}}\left[\mathcal{T}_{s}\left(r_{s}, t_{s}\right) \int \frac{1}{r^{\prime}} f_{s}\left(x, r^{\prime}, t_{s}\right) d r^{\prime}-\frac{1}{r_{s}} f_{s}\left(x, r_{s}, t_{s}\right)\right] \text {, }
$$

$$
\text { (b) } f_{s}(x, r, 0)=0, \quad\left(\text { c) } f_{s}\left(0, r, t_{s}\right)=r \lambda_{s}\left(t_{s}\right) \mathcal{T}_{s}\left(r, t_{s}\right)\right. \text {. }
$$

The flux $F$ is now for $\varepsilon \ll 1$ expressed in terms of the density $\rho$ via a functional expansion, namely the Chapman-Enskog expansion [4]. We have the following theorem.

THEOREM T3. Let $T_{-1}(t), T_{1}(t)$, and $T_{2}(t)$ denote the moments of the probability distribution $\mathcal{T}(r, t)$, i.e., $T_{j}(t)=\int r^{j} \mathcal{T}(r, t) d r, j=-1,1,2$. For the dimensionless parameter $\varepsilon \ll 1$ the flux $F$ in (4.2) is given in terms of the density $\rho$ as

$$
F(x, t)=C(t) \rho(x, t)-D(t) \partial_{x} \rho(x, t)+O\left(\varepsilon^{2}\right)
$$


with the convection coefficient $C$ and the diffusion coefficient $D$ given by

$$
C=\frac{1}{T_{1}}+\frac{\varepsilon T_{-1}}{\lambda} \frac{1}{T_{1}} \partial_{t} \frac{T_{2}}{T_{1}}, \quad D=\frac{\varepsilon T_{-1}}{\lambda} \frac{T_{2}-T_{1}^{2}}{T_{1}^{3}} .
$$

The proof of Theorem T3 is deferred to section 7 .

Reversing the scaling from above we obtain the initial boundary value problem

$$
\text { (a) } \partial_{t} \rho+\partial_{x} F=0, \quad x, t>0, \quad F(x, t)=C(t) \rho-D(t) \partial_{x} \rho,
$$

(b) $F(0, t)=\lambda(t), \quad \rho(x, 0)=0, \quad\left(\right.$ c) $C=\frac{1}{T_{1}}+\frac{T_{-1}}{\lambda} \frac{1}{T_{1}} \partial_{t} \frac{T_{2}}{T_{1}}, \quad D=\frac{T_{-1}}{\lambda} \frac{T_{2}-T_{1}^{2}}{T_{1}^{3}}$

in unscaled variables.

Remark. The diffusion coefficient $D(t)$ in (4.6) is guaranteed to be nonnegative, since the term $T_{2}-\left(T_{1}\right)^{2}$ represents the variance of the distribution $\mathcal{T}$.

Remark. For a time-independent distribution $\mathcal{T}(r, t)=\mathcal{T}(r)$ and a constant influx density $\lambda$, the steady states of (4.8) which are bounded at $x=\infty$ are obviously given by spatially constant solutions satisfying $\lambda=C \rho$. Since, for spatially constant $\rho$, the value of $\rho$ coincides with the WIP $W(t)$, and since in the time-independent case $C=\frac{1}{T_{1}}$ holds, we obtain the relation $W=\lambda T_{1}$. Thus we recover, up to a scaling factor introduced by using arrival probabilities instead of the arrival density, the well-known Little's law $W=\lambda \tau$ [12], [7] for the steady-state situation.

Remark. Other than in classical gas dynamics, the equilibrium distribution $r \mathcal{T}(r, t)$, the equivalent of the Maxwellian, is time-dependent in our case. This results in the correction to the convection coefficient $C$ in (4.8)(c).

5. Numerical results. In this section we carry out a numerical experiment to illustrate the difference between the discrete event algorithm (1.1) on one hand and the random phase approach $(3.1),(4.8)$ on the other hand [11]. This experiment is supposed to demonstrate the following features.

- While the random phase model and the discrete event model should give the same results for the steady state, the random phase model is expected to react more rapidly to changes in the TPT than the discrete event model, thus reflecting the reentrant nature of the factory.

- In Theorem T1 we have made a mean field assumption, namely, that the probability distribution $\mathcal{T}(r, t)$ for the TPT in (2.4) is a given function, while in reality it depends on the WIP $W(t)$. The justification for this assumption is that if many lots are considered the impact of one individual lot on the WIP $W$ is negligible. The Boltzmann equation (3.5) and its diffusion approximation (4.8) have both been derived under this assumption. For the numerical experiment in this section we use a WIP-dependent distribution $\mathcal{T}=\mathcal{T}(r, W(t))$, and so comparing solutions of the particle model (3.1) with solutions of the diffusion equation (4.8) serves also to justify the mean field assumption.

- We numerically verify the validity of the Chapman-Enskog approximation in section 4, which has been derived only formally.

Experimental data usually come in the form of a mean TPT dependent on the WIP $W(t)$, a maximal observed TPT, and a minimal observed TPT. We choose a distribution whose mean is a function of the WIP. The maximal possible TPT is a constant multiple of the mean, while the minimally possible TPT remains constant, 




FIG. 5.1. Mean, upper, and lower limits of $\mathcal{T}(r, W)$.

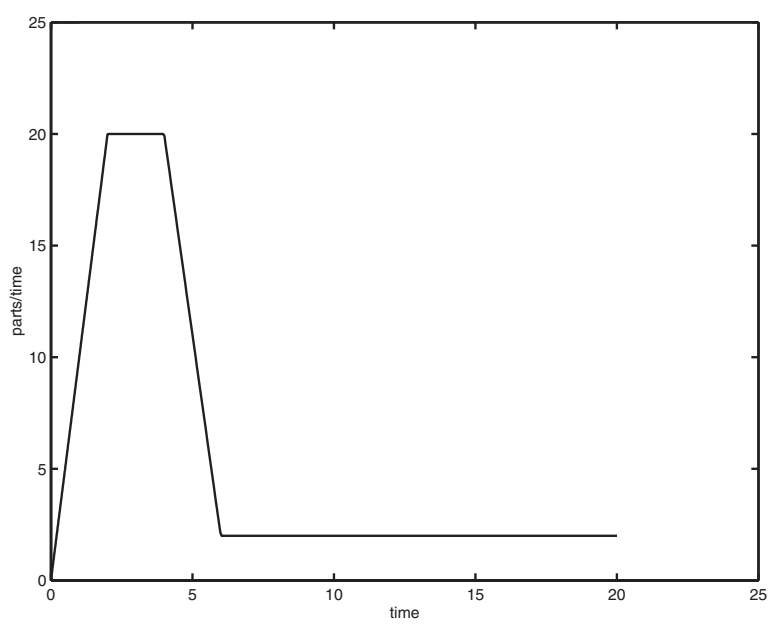

Fig. 5.2. Influx.

independent of the WIP. This is consistent with the experimental observation that, regardless of the load, there will always be "fast" lots which pass through the factory almost unperturbed by the others. We choose a distribution which is constant between the mean and the upper and lower limit, respectively. Figure 5.1 shows the upper and lower limits as well as the mean as a function of WIP. Figure 5.2 shows the influx $\lambda(t)$ used in the experiment.

We first compare the results of the discrete event algorithm (1.1), which we refer to as the queuing model, to the random phase model. We compute the trajectories given by the random phase model (3.1) for $N=400$ lots with $N$ deterministic arrival times $a_{n}$, given by

$$
a_{n+1}=a_{n}+\frac{1}{\lambda\left(a_{n}\right)}, \quad a_{0}=0,
$$

and compare them to the corresponding solutions of (3.6) for the queuing model 


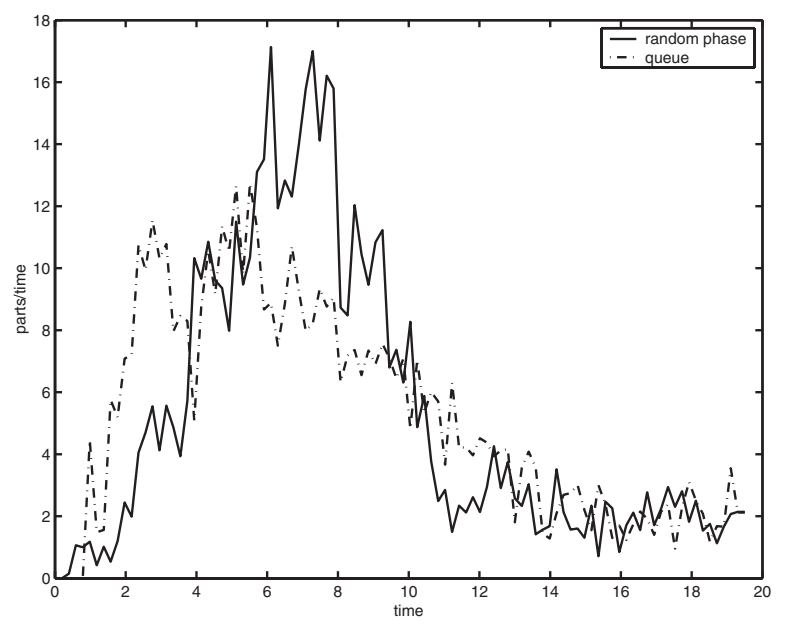

Fig. 5.3. Outflux (random phase and queue); $N=400$ lots, 20 ensembles.

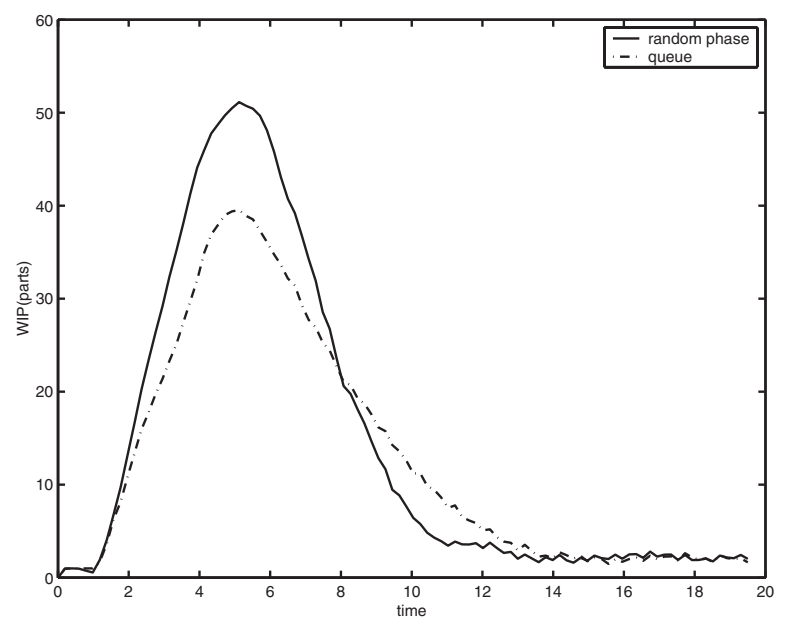

FIG. 5.4. WIP $W(t) ; N=400$ lots, 20 ensembles.

trajectories. Figure 5.3 shows the outflux for both models, and Figures 5.4 and 5.5 show the resulting WIP $W(t)$, computed according to (3.2), as well as the expectation of the TPT $\int r \mathcal{T}(r, W(t)) d r$ for both models. We observe that, roughly, between $t=2$ and $t=4$ the outflux of the queuing model is larger since the random phase model has already reacted to the increased TPT. Between $t=4$ and $t=8$ the outflux of the random phase model is larger, since the increased influx now exits at a later time. For $t>12$ we have reached a steady state, where the queuing model and the random phase model are equivalent because of our choice of the update frequency $\omega(r, t)$ in (3.7). Using individual lot trajectories, the fluxes, computed according to (3.2) with discrete arrival times $a_{n}$, are a superposition of $\delta$-functions. To depict them, they have to be smoothed, i.e., convoluted, with an indicator function. Even after applying this smoothing procedure, the signal-to-noise ratio in these computations is rather large, so Figures 5.3, 5.4, and 5.5 have been obtained by running the simulation 20 times and computing averages. 


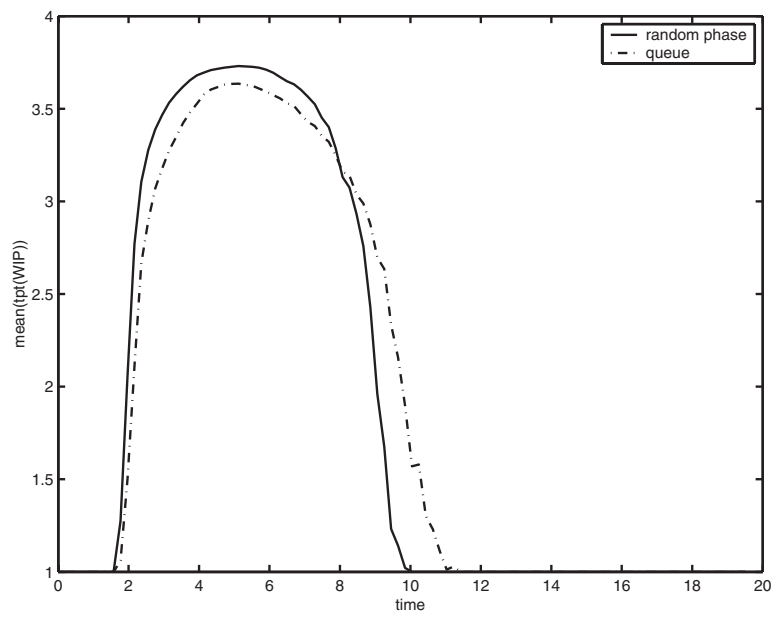

FIG. 5.5. Mean TPT $T_{1}(W(t)) ; N=400$ lots, 20 ensembles.



FIG. 5.6. Outflux for the random phase model and the diffusion equation; $N=400$ lots, 20 ensembles.

Next we compare the averaged flux computed from the random phase model (2.4), i.e., the expectations, to the solution of the diffusion equation (4.2) with the constitutive law for the flux $F$ given by (4.6); i.e., we verify the validity of the ChapmanEnskog expansion. The diffusion equation (4.2) is given on the half line $x>0$, while we are interested only in the solution $\rho$ for $0<x<1$ and the flux $F$ at $x=1$. Thus some kind of numerical radiation condition has to be imposed to truncate the infinite computational domain. We discretized convection term $\partial_{x} C \rho$ in the diffusion equation (4.2) by upwind differences and the diffusion term $-\partial_{x} D \partial_{x} \rho$ by centered differences, setting the diffusion coefficient $D(t)$ to zero, sufficiently far out (at $x=5$; doubling the computational domain did not produce a visible difference in the solution). Figure 5.6 shows the comparison of the fluxes for the random phase flux model and the diffusion equation, which agree up to expected random fluctuations.

A key parameter in the simulation via the diffusion equation (4.2) is the coefficient 


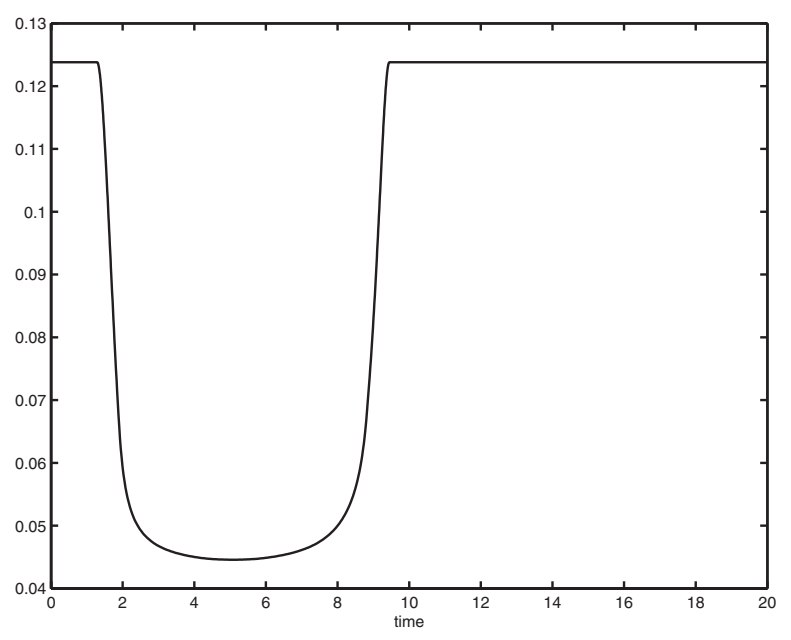

FIG. 5.7. Ratio of diffusion to convection coefficient.

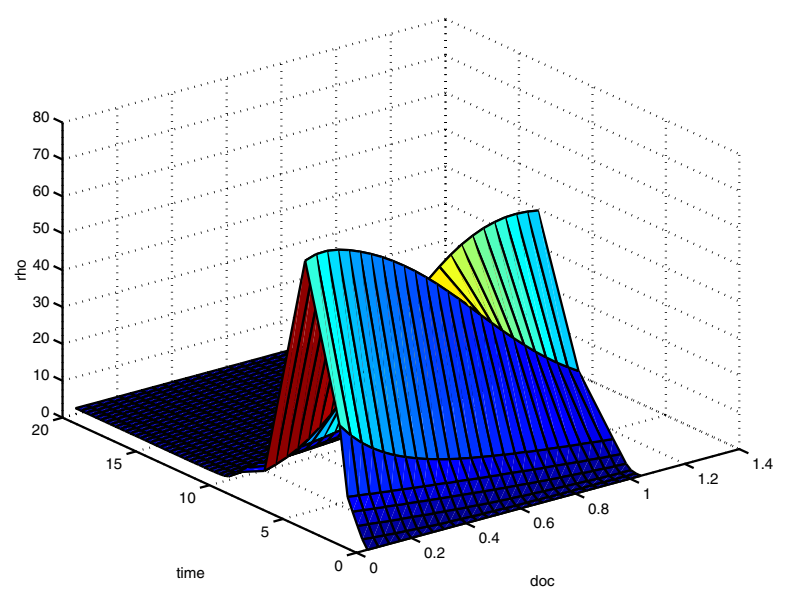

FIG. 5.8. Part density $\rho$.

of variation, i.e., the ratio $\frac{C}{D}=\frac{T_{2}-\left(T_{1}\right)^{2}}{\omega_{0}\left(T_{1}\right)^{2}}$ of diffusion to convection, giving the stochastic contribution to the diffusion equation model. Figure 5.7 shows this ratio as a function of time. For the coefficient of variation identically equal to zero (pure convection) the differential equation would reduce to the simple model discussed in [2]. Figure 5.7 shows that the variance of the TPT distribution contributes significantly to the structure of the solution in the transient phase. Finally, Figure 5.8 depicts the density $\rho$ as a function of DOC and time.

6. Conclusions. In order to account for dynamic changes in the WIP during the processing time of a lot in a reentrant supply chain node, we have introduced the concept of a random phase velocity. In the context of gas dynamical and fluid dynamical models for supply chains, this corresponds to introducing the concept of collisions, temperature, and diffusion. The temperature is defined as a combination of the variance of the estimated TPT distribution and the frequency with which the TPT is updated. This allows for extending the zero temperature moment closure 
sytems, presented in [3], to simpler convection-diffusion equations. The resulting models exhibit a more immediate response to steep ramps in the product influx than standard discrete event models corresponding to zero temperature moment closures.

\section{Appendix.}

Proof of Theorem T1. The proof consists of essentially summing up all possibilities for the values of $\kappa(t), \xi_{a}(t)$, and $\eta_{a}(t)$ and weighting them with their respective probabilities. This takes the form

$$
\begin{aligned}
& f_{a}(x, r, t+\Delta t) \\
& =\int \delta\left(x^{\prime}+\frac{\Delta t}{r^{\prime}}-x\right) \delta\left(k u+(1-k) r^{\prime}-r\right) \\
& \quad \times d \mathcal{P}\{\kappa(t)=k\} d \mathcal{P}\{\tau(t)=u\} d \mathcal{P}\left\{\xi_{a}(t)=x^{\prime}, \eta_{a}(t)=r^{\prime}\right\} \\
& =\int \delta\left(x^{\prime}+\frac{\Delta t}{r^{\prime}}-x\right) \delta\left(k u+(1-k) r^{\prime}-r\right) \\
& \quad \times\left[\Delta t \omega\left(r^{\prime}, t\right) \delta(k-1)+\left(1-\Delta t \omega\left(r^{\prime}, t\right)\right) \delta(k)\right] \mathcal{T}(u, t) f_{a}\left(x^{\prime}, r^{\prime}, t\right) d s^{\prime} d r^{\prime} d u d k .
\end{aligned}
$$

Integrating the $\delta$-functions with respect to $k$ gives

$$
\begin{gathered}
f_{a}(x, r, t+\Delta t)=\int \Delta t \omega\left(r^{\prime}, t\right) \delta\left(x^{\prime}+\frac{\Delta t}{r^{\prime}}-x\right) \delta(u-r) \mathcal{T}(u, t) f_{a}\left(x^{\prime}, r^{\prime}, t\right) d s^{\prime} d r^{\prime} d u \\
+\int\left(1-\Delta t \omega\left(r^{\prime}, t\right)\right) \delta\left(x^{\prime}+\frac{\Delta t}{r^{\prime}}-x\right) \delta\left(r^{\prime}-r\right) \mathcal{T}(u, t) f_{a}\left(x^{\prime}, r^{\prime}, t\right) d s^{\prime} d r^{\prime} d u
\end{gathered}
$$

and integrating with respect to $x^{\prime}, r^{\prime}$, and $u$ in the above two integrals gives

$$
\begin{aligned}
f_{a}(x, r, t+\Delta t)= & \int \Delta t \omega\left(r^{\prime}, t\right) \mathcal{T}(r, t) f_{a}\left(x-\frac{\Delta t}{r^{\prime}}, r^{\prime}, t\right) d r^{\prime} \\
& +(1-\Delta t \omega(r, t)) f_{a}\left(x-\frac{\Delta t}{r}, r, t\right)
\end{aligned}
$$

where we have used the fact that $\int \mathcal{T}(u, t) d u=1$ holds. We now multiply (7.1) by a test function $\psi(x, r, t)$ and integrate over $t>a$, giving

$$
\begin{aligned}
& \int H(t-a) \psi(x, r, t) f_{a}(x, r, t+\Delta t) d x d r d t \\
& =\int H(t-a) \psi(x, r, t) \Delta t \omega\left(r^{\prime}, t\right) \mathcal{T}(r, t) f_{a}\left(x-\frac{\Delta t}{r^{\prime}}, r^{\prime}, t\right) d r^{\prime} d x d r d t \\
& \quad+\int H(t-a) \psi(x, r, t)(1-\Delta t \omega(r, t)) f_{a}\left(x-\frac{\Delta t}{r}, r, t\right) d x d r d t,
\end{aligned}
$$

where $H$ denotes the Heaviside function. Shifting the arguments in the integrals gives

$$
\begin{aligned}
& \int H(t-\Delta t-a) \psi(x, r, t-\Delta t) f_{a}(x, r, t) d x d r d t \\
& =\int H(t-a) \psi\left(x+\frac{\Delta t}{r^{\prime}}, r, t\right) \Delta t \omega\left(r^{\prime}, t\right) \mathcal{T}(r, t) f_{a}\left(x, r^{\prime}, t\right) d r^{\prime} d x d r d t \\
& \quad+\int H(t-a) \psi\left(x+\frac{\Delta t}{r}, r, t\right)(1-\Delta t \omega(r, t)) f_{a}(x, r, t) d x d r d t .
\end{aligned}
$$


The left-hand side of the above equation can be rewritten as

$$
\begin{aligned}
& \int H(t-a) \psi(x, r, t-\Delta t) f_{a}(x, r, t) d x d r d t-\int_{a}^{a+\Delta t}\left[\int \psi(x, r, t-\Delta t) f_{a}(x, r, t) d x d r\right] d t \\
& =\int H(t-a)\left[\psi-\Delta t \partial_{t} \psi\right] f_{a}(x, r, t) d x d r d t-\Delta t \int \psi(x, r, a) f_{a}(x, r, a) d x d r+O\left(\Delta t^{2}\right) \\
& =\int H(t-a)\left[\psi-\Delta t \partial_{t} \psi\right] f_{a}(x, r, t) d x d r d t-\Delta t \int \psi(0, r, a) \mathcal{T}(r, a) d r+O\left(\Delta t^{2}\right) .
\end{aligned}
$$

Expanding the right-hand side of (7.2) gives

$$
\begin{aligned}
& \int H(t-a) \psi(x, r, t) \Delta t \omega\left(r^{\prime}, t\right) \mathcal{T}(r, t) f_{a}\left(x, r^{\prime}, t\right) d r^{\prime} d x d r d t \\
& +\int H(t-a)\left(\psi+\frac{\Delta t}{r} \partial_{x} \psi\right) f_{a}(x, r, t) d x d r d t \\
& -\int H(t-a) \psi(x, r, t) \Delta t \omega(r, t) f_{a}(x, r, t) d x d r d t+O\left(\Delta t^{2}\right) .
\end{aligned}
$$

Setting this equal to (7.3) and dividing by $\Delta t$ gives

$$
\begin{aligned}
& -\int H(t-a)\left[\partial_{t} \psi+\frac{1}{r} \partial_{x} \psi\right] f_{a}(x, r, t) d x d r d t-\int \psi(0, r, a) \mathcal{T}(r, a) d r \\
& =\int H(t-a) \psi(x, r, t) \omega\left(r^{\prime}, t\right) \mathcal{T}(r, t) f_{a}\left(x, r^{\prime}, t\right) d r^{\prime} d x d r d t \\
& \quad-\int H(t-a) \psi(x, r, t) \omega(r, t) f_{a}(x, r, t) d x d r d t+O(\Delta t),
\end{aligned}
$$

which for $\Delta t \rightarrow 0$ is the weak formulation of (3.3).

Proof of Theorem T2. The weak form of the initial value problem (3.3) implies that for any test function $\psi(x, r, t)$ the relation

$$
+\int\left\{H(t-a) \psi(x, r, t)\left[\mathcal{T}(r, t) \int \omega\left(r^{\prime}, t\right) f_{a}\left(x, r^{\prime}, t\right) d r^{\prime}-\omega(r, t) f_{a}(x, r, t)\right]\right\} d x d r d t
$$

holds, where all integrals range over $\mathbb{R}$ and $H$ denotes the usual Heaviside function. Multiplying (7.4) by $\lambda(a)$ and integrating with respect to $a$ gives

$$
\begin{gathered}
-\int f\left[\partial_{t} \psi+\frac{1}{r} \partial_{x} \psi\right](x, r, t) d x d r d t=\int \psi(0, r, a) \mathcal{T}(r, a) \lambda(a) d a d r \\
+\int\left\{\psi(x, r, t)\left[\mathcal{T}(r, t) \int \omega\left(r^{\prime}, t\right) f\left(x, r^{\prime}, t\right) d r^{\prime}-\omega(r, t) f(x, r, t)\right]\right\} d x d r d t .
\end{gathered}
$$

Now $f_{a}(x, r, t)$ vanishes identically for $x<0$ and $\lambda(a)$ vanishes identically for $a<0^{\prime}$. Therefore $f(x, r, t)$ vanishes identically for $x<0, t<0$. Thus $(7.5)$ is the same as

$$
\begin{aligned}
& -\int H(x) H(t) f\left[\partial_{t} \psi+\frac{1}{r} \partial_{x} \psi\right](x, r, t) d x d r d t=\int H(t) \psi(0, r, t) \mathcal{T}(r, t) \lambda(t) d t d r \\
& +\int\left\{H(x) H(t) \psi(x, r, t)\left[\mathcal{T}(r, t) \int \omega\left(r^{\prime}, t\right) f\left(x, r^{\prime}, t\right) d r^{\prime}-\omega(r, t) f(x, r, t)\right]\right\} d x d r d t .
\end{aligned}
$$


Equation (7.6) is the weak formulation of the boundary value problem (3.5).

Proof of Theorem T3. The Chapman-Enskog expansion consists of deriving a functional dependence of the kinetic density $f$ on the fluid density $\rho$ of the form

$$
f(x, r, t)=\Psi(\rho(x, t), r, t),
$$

where for any $\rho$ the shape function $\Psi$ has to satisfy

$$
\int \Psi(\rho, r, t)=\rho \quad \forall \rho .
$$

Given the shape function $\Psi$ the flux $F$ is then computed as $F(\rho, x, t)=\int \frac{1}{r} \Psi(\rho, r, t) d r$. Inserting (7.7) into the scaled Boltzmann equation (4.5)(a) gives

$$
\begin{gathered}
-D_{\rho} \Psi \partial_{x} F+\partial_{t} \Psi+\frac{1}{r} \partial_{x} \Psi=\frac{\lambda}{\varepsilon T_{-1}} Q[\Psi], \\
Q[\Psi]:=\mathcal{T}(r, t) \int \frac{1}{r^{\prime}} \Psi\left(\rho, r^{\prime}, t\right) d r^{\prime}-\frac{1}{r} \Psi(\rho, r, t) .
\end{gathered}
$$

Here we write $D_{\rho} \Psi$ for the derivative of $\Psi$ with respect to $\rho$ since $\Psi$ will depend in general also on the derivatives of the density $\rho$; i.e., $D_{\rho}$ denotes a Frechet derivative. The shape function $\Psi$ is now obtained by solving (7.9) asymptotically for $\varepsilon \ll 1$ through an expansion of the form $\Psi=\Psi_{0}+\varepsilon \Psi_{1}+\cdots$. To compute the terms in this expansion we will need a Fredholm alternative for the operator $Q$ in (7.9). Direct calculus gives that the equation $Q[f](x, r, t)=g(x, r, t)$ has a solution if and only if $\int g d r=0$ for all $x, t$ holds. In this case the solution is given by

$$
f(x, r, t)=r[c(x, t) \mathcal{T}(r, t)-g(x, r, t)]
$$

for an arbitrary function $c(x, t)$. For the zero-order term $\Psi_{0}$ we obtain

$$
Q\left[\Psi_{0}\right]=0 \quad \Rightarrow \quad \Psi_{0}(\rho, r, t)=\frac{\rho r \mathcal{T}(r, t)}{T_{1}(t)},
$$

where the normalization constant $c$ in (7.10) is obtained from the condition (7.8). This gives in zeroth order for the flux $F$

$$
F_{0}(\rho, x, t)=\int \frac{1}{r} \Psi_{0}(\rho, r, t) d r=\frac{\rho}{T_{1}} .
$$

Going to the $O(\varepsilon)$ term in $(7.9)$ gives

$$
-D_{\rho} \Psi_{0} \partial_{x} F_{0}+\partial_{t} \Psi_{0}+\frac{1}{r} \partial_{x} \Psi_{0}=\frac{\lambda}{T_{-1}} Q\left[\Psi_{1}\right]
$$

or

$$
\frac{T_{-1}}{\lambda}\left[\rho r \partial_{t} \frac{\mathcal{T}}{T_{1}}+\frac{\mathcal{T}}{T_{1}^{2}}\left(T_{1}-r\right) \partial_{x} \rho\right]=Q\left[\Psi_{1}\right]
$$

The left-hand side of the equation vanishes when integrating with respect to $r$, and (7.10) gives

$$
\Psi_{1}=c(x, t) r \mathcal{T}(r, t)-\frac{T_{-1}}{\lambda}\left[\rho r^{2} \partial_{t} \frac{\mathcal{T}}{T_{1}}+\frac{\mathcal{T}}{T_{1}^{2}}\left(T_{1} r-r^{2}\right) \partial_{x} \rho\right]
$$


for an arbitrary function $c(x, t)$. This function is again determined by the normalization condition (7.8), which implies that $\int \Psi_{1}(\rho, r, t) d r=0$ for all $\rho, t$ has to hold. This gives for $c$

$$
c(x, t)=\frac{T_{-1}}{\lambda}\left[\frac{\rho}{T_{1}} \partial_{t} \frac{T_{2}}{T_{1}}+\frac{T_{1}^{2}-T_{2}}{T_{1}^{3}} \partial_{x} \rho\right], \quad T_{2}(t):=\int r^{2} \mathcal{T}(r, t) d r,
$$

and for the first-order term in $\Psi$

$$
\Psi_{1}=\frac{T_{-1}}{\lambda}\left[\frac{\rho r \mathcal{T}}{T_{1}} \partial_{t} \frac{T_{2}}{T_{1}}-\rho r^{2} \partial_{t} \frac{\mathcal{T}}{T_{1}}+r \mathcal{T} \frac{T_{1}^{2}-T_{2}}{T_{1}^{3}} \partial_{x} \rho-\frac{\mathcal{T}}{T_{1}^{2}}\left(T_{1} r-r^{2}\right) \partial_{x} \rho\right] .
$$

Finally, we obtain for the first-order term in the flux

$$
F_{1}=\int \frac{1}{r} \Psi_{1} d r=\frac{T_{-1}}{\lambda}\left[\frac{\rho}{T_{1}} \partial_{t} \frac{T_{2}}{T_{1}}+\frac{T_{1}^{2}-T_{2}}{T_{1}^{3}} \partial_{x} \rho\right] .
$$

So, altogether, the flux is given in terms of $\rho$ up to order $O\left(\varepsilon^{2}\right)$ terms by

$$
F_{0}+\varepsilon F_{1}=C(t) \rho-D(t) \partial_{x} \rho
$$

with the convection coefficient $C$ and the diffusion coefficient $D$ given by

$$
C=\frac{1}{T_{1}}+\frac{\varepsilon T_{-1}}{\lambda} \frac{1}{T_{1}} \partial_{t} \frac{T_{2}}{T_{1}}, \quad D=\frac{\varepsilon T_{-1}}{\lambda} \frac{T_{2}-T_{1}^{2}}{T_{1}^{3}} .
$$

Reversing the scaling, we obtain the same initial boundary value problem as (4.8) with the convection and diffusion coefficients $C$ and $D$ given by

$$
C=\frac{1}{T_{1}}+\frac{T_{-1}}{\lambda} \frac{1}{T_{1}} \partial_{t} \frac{T_{2}}{T_{1}}, \quad D=\frac{T_{-1}}{\lambda} \frac{T_{2}-T_{1}^{2}}{T_{1}^{3}},
$$

where now $T_{1}$ and $T_{2}$ denote the first and second moments of the original distribution $\mathcal{T}$.

\section{REFERENCES}

[1] E. J. Anderson, A new continuous model for job shop scheduling, Internat. J. Systems Sci., 12 (1981), pp. 1469-1475.

[2] D. Armbruster, D. Marthaler, C. Ringhofer, K. Kempf, and T.-C. Jo, A continuum model for a re-entrant factory, Oper. Res., submitted.

[3] D. Armbruster, D. Marthaler, and C. Ringhofer, Kinetic and fluid model hierarchies for supply chains, Multiscale Model. Simul., 2 (2004), pp. 43-61.

[4] S. G. Brush, Kinetic Theory, Pergamon Press, Oxford, UK, 1972.

[5] C. Cercignani, The Boltzmann Equation and Its Applications, Appl. Math. Sci. 67, SpringerVerlag, New York, 1988.

[6] C. Daganzo, A Theory of Supply Chains, Lecture Notes in Econom. and Math. Systems 526, Springer-Verlag, Berlin, 2003.

[7] M. el-Taha and S. Stidham, Sample-Path Analysis of Queuing Systems, Internat. Ser. Oper. Res. Management Sci. 11, Kluwer Academic Publishers, Boston, MA, 1999.

[8] J. Hasenbein, Stability of fluid networks with proportional routing, Queueing Syst., 38 (2001), pp. 327-354.

[9] D. Helbing, Gas kinetic derivation of Navier Stokes like traffic equations, Phys. Rev. E (3), 53 (1996), pp. 2366-2381.

[10] D. Helbing, Traffic and related self-driven many particle systems, Rev. Modern Phys., 73 (2001), pp. 1067-1141.

[11] R. W. Hockney and J. W. Eastwood, Computer Simulation Using Particles, McGraw-Hill, Maidenhead, UK, 1981.

[12] J. D. C. LitTle, A proof of the queueing formula $L=\lambda W$, Oper. Res., 9 (1961), pp. 383-387.

[13] S. H. Lu ANd P. R. Kumar, Distributed scheduling based on due dates and buffer priorities, IEEE Trans. Automat. Control, 36 (1991), pp. 1406-1416. 